[bmim+]

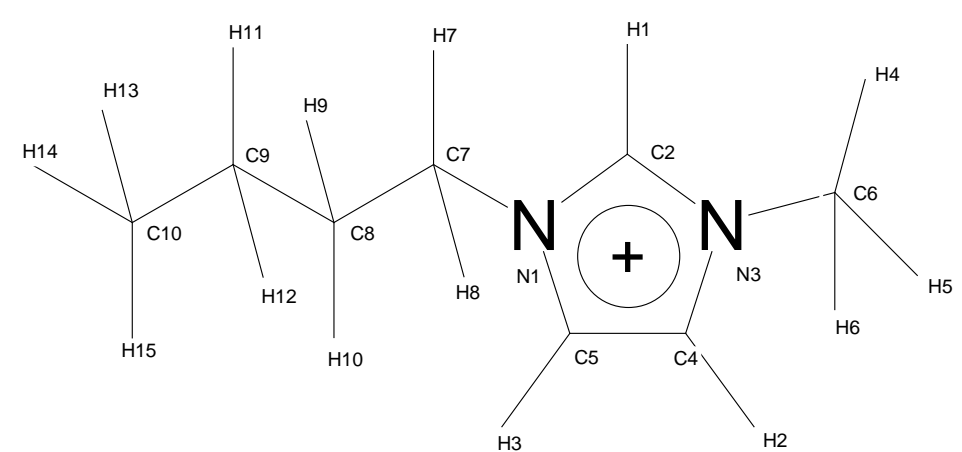

[bmmim+]

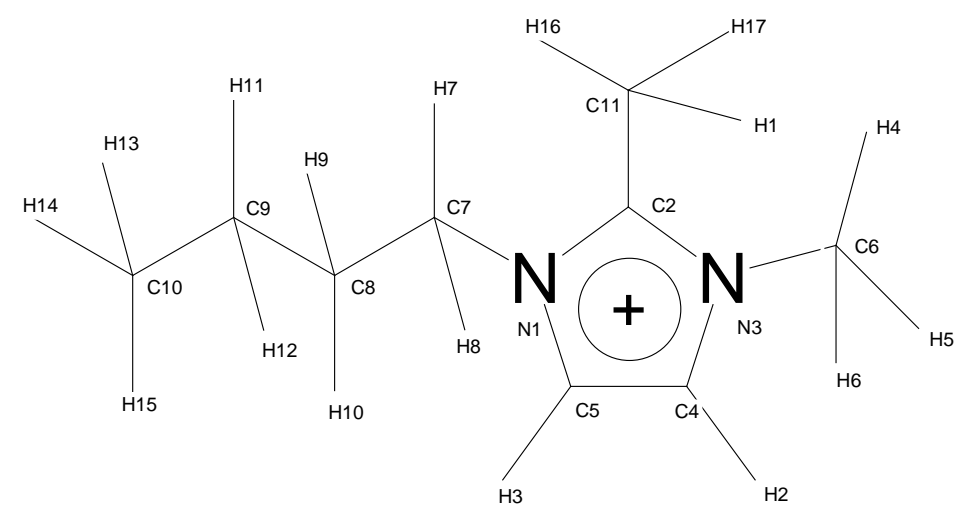

$\mathrm{PF}_{6}$

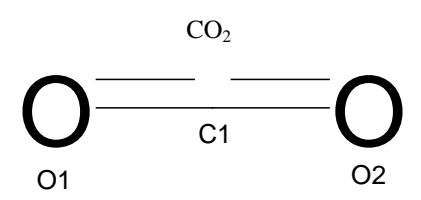

[bmim] $\left[\mathrm{PF}_{6}\right]$

Partial Atomic Charges and Lennard-Jones Parameters

[bmmim] $\left[\mathrm{PF}_{6}\right]$

Partial Atomic Charges and Lennard-Jones Parameters

\begin{tabular}{lllll|lllll}
\hline Atom ID & Type & $\mathrm{q}_{\mathrm{i}}(\mathrm{e})$ & $\sigma(\mathrm{A})$ & $\varepsilon_{i}\left(\mathrm{kcal} \mathrm{mol}^{-1}\right)$ & Atom ID & Type & $\mathrm{q}_{i}(\mathrm{e})$ & $\sigma(\mathrm{A})$ & $\varepsilon_{i}\left(\mathrm{kcal} \mathrm{mol}^{-1}\right)$ \\
\hline $\mathrm{C}_{4}$ & $\mathrm{CPH} 1$ & -0.170284 & 3.207 & 0.0500 & $\mathrm{C}_{4}$ & $\mathrm{CPH} 1$ & -0.215065 & 3.207 & 0.0500
\end{tabular}

$\begin{array}{llllllllll}\mathrm{C}_{4} & \mathrm{CPH} 1 & -0.170284 & 3.207 & 0.0500 & \mathrm{C}_{4} & \mathrm{CPH} 1 & -0.215065 & 3.207 & 0.0500 \\ \mathrm{C}_{5} & \mathrm{CPH} 1 & -0.205659 & 3.207 & 0.0500 & \mathrm{C}_{5} & \mathrm{CPH} 1 & -0.103616 & 3.207 & 0.0500 \\ \mathrm{~N}_{1} & \mathrm{NR1} & 0.209886 & 3.296 & 0.000 & \mathrm{~N}_{2} & \mathrm{NH} & -0.023997 & 3.296 & 0.2000\end{array}$

$\begin{array}{llllllllll}\mathrm{C}_{5} & \mathrm{CPH} 1 & -0.205659 & 3.207 & 0.0500 & \mathrm{C}_{5} & \mathrm{CPH} 1 & -0.103616 & 3.207 & 0.0500 \\ \mathrm{~N}_{1} & \mathrm{NR} 1 & 0.209886 & 3.296 & 0.2000 & \mathrm{~N}_{1} & \mathrm{NR} 1 & -0.023997 & 3296 & 0.2000 \\ \mathrm{C}_{2} & \mathrm{CPH} & -0.10655 & 3.207 & 0.0500 & \mathrm{C}_{2} & \mathrm{CPH} & 0.253950 & 3.207 & 0.0500\end{array}$

\begin{tabular}{lllll|llllll}
$\mathrm{N}_{1}$ & $\mathrm{NR} 1$ & 0.209886 & 3.296 & 0.2000 & $\mathrm{~N}_{1}$ & $\mathrm{NR} 1$ & -0.023997 & 3.296 & 0.2000 \\
$\mathrm{C}^{2}$ & $\mathrm{CPH}$ & 0.106455 & 3.207 & 0.0500 & $\mathrm{C}_{2}$ & $\mathrm{CPH}$ & 0.253950 & 3.207 & 0.0500
\end{tabular}

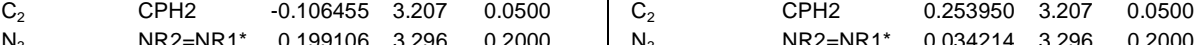

\begin{tabular}{lllll|lllll}
$\mathrm{N}_{3}$ & $\mathrm{NR} 2=\mathrm{NR} 1$ & 0.199106 & 3.296 & 0.2000 & $\mathrm{~N}_{3}$ & $\mathrm{NR} 2=\mathrm{NR} 1$ & 0.034214 & 3.296 & 0.2000 \\
$\mathrm{H}_{2}$ & $\mathrm{HR} 3$ & 0.184617 & 2.616 & 0.0078 & $\mathrm{H}_{2}$ & $\mathrm{HR} 3$ & 0.217487 & 2.616 & 0.0078
\end{tabular}

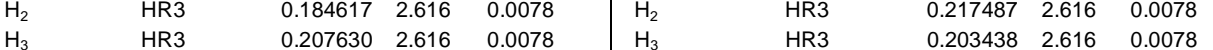

$\begin{array}{llllllllll}\mathrm{H}_{3} & \mathrm{HR} 3 & 0.207630 & 2.616 & 0.0078 & \mathrm{H}_{3} & \mathrm{HR} 3 & 0.203438 & 2.616 & 0.0078 \\ \mathrm{H} & \mathrm{HR} 1 & 0.223622 & 1.604 & 0.0460 & \mathrm{C}_{6} & \mathrm{CN7B} & -0.089528 & 4.054 & 0.0200\end{array}$

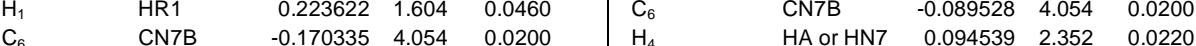

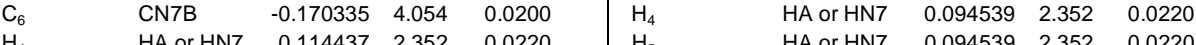

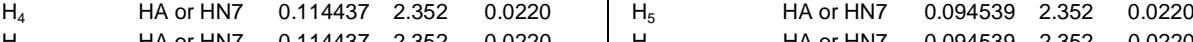

\begin{tabular}{lllll|lllll}
$\mathrm{H}_{5}$ & $\mathrm{HA}$ or HN7 & 0.114437 & 2.352 & 0.0220 & $\mathrm{H}_{6}$ & $\mathrm{HA}$ or HN7 & 0.094539 & 2.352 & 0.0220
\end{tabular}

$\begin{array}{lllllllllll}\mathrm{H}_{6} & \mathrm{HA} \text { or HN7 } & 0.114437 & 2.352 & 0.0220 & \mathrm{C}_{7} & \text { CN7B } & 0.046706 & 4.054 & 0.0200\end{array}$

$\begin{array}{lllllllllll}\mathrm{C}_{7} & \mathrm{CN7B} & -0.116193 & 4.054 & 0.0200 & \mathrm{H}_{7} & \mathrm{HA} \text { or HN7 } & 0.068196 & 2.352 & 0.0220 \\ \mathrm{H}_{7} & \mathrm{HA} \mathrm{or} \mathrm{HN7} & 0.087944 & 2.352 & 0.0220 & \mathrm{H}_{8} & \text { HA or HN7 } & 0.068196 & 2.352 & 0.0220\end{array}$

\begin{tabular}{lllll|llllll}
$\mathrm{H}_{8}$ & $\mathrm{HA}$ or HN7 & 0.087944 & 2.352 & 0.0220 & $\mathrm{C}_{8}$ & $\mathrm{CT} 2$ & -0.023644 & 3.875 & 0.0550
\end{tabular}

$\begin{array}{llllllllll}\mathrm{C}_{8} & \mathrm{CT} 2 & 0.085859 & 3.875 & 0.0550 & \mathrm{H}_{9} & \mathrm{HA} & -0.002247 & 2.352 & 0.0220\end{array}$

$\begin{array}{llllllllll}\mathrm{H}_{9} & \mathrm{HA} & -0.023431 & 2.352 & 0.0220 & \mathrm{H}_{10} & \mathrm{HA} & -0.002247 & 2.352 & 0.0220\end{array}$

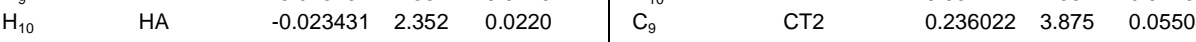

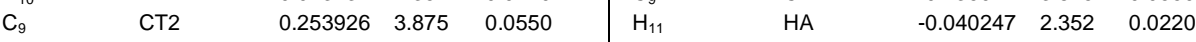

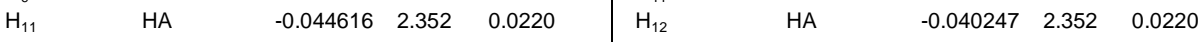

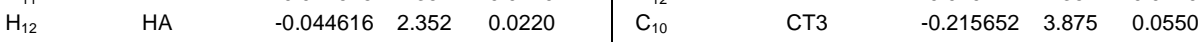

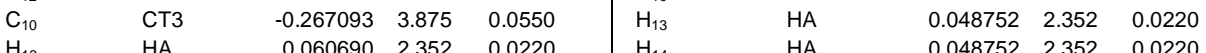

$\begin{array}{llllllllll}\mathrm{H}_{13} & \mathrm{HA} & 0.060690 & 2.352 & 0.0220 & \mathrm{H}_{14} & \mathrm{HA} & 0.048752 & 2.352 & 0.0220 \\ \mathrm{H}_{14} & \mathrm{HA} & 0.060690 & 2.352 & 0.0220 & \mathrm{H}_{15} & \mathrm{HA} & 0.048752 & 2352 & 0.0220 \\ \mathrm{H}_{15} & \mathrm{HA} & 0.06069 & 2352 & 0.0220 & \mathrm{C}_{11} & \mathrm{CN} & -0.29069 & 3.635 & 0.0780\end{array}$

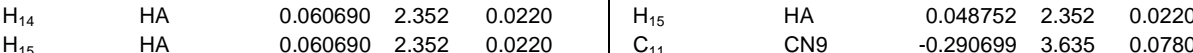

$\begin{array}{lllllll} & & \mathrm{H}_{1} & \mathrm{HN} 9 & 0.124579 & 2.388 & 0.0240\end{array}$

\begin{tabular}{llll|lllll}
$\mathrm{P}$ & 1.433886 & 3.831 & 0.5850 & $\mathrm{H}_{16}$ & $\mathrm{HN9}$ & 0.124579 & 2.388 & 0.0240
\end{tabular}

\begin{tabular}{llll|llllll} 
FN1 & -0.387948 & 3.029 & 0.0900 & $H_{17}$ & HN9 & 0.124579 & 2.388 & 0.0240
\end{tabular}

$\begin{array}{llll}\text { FN2 } & -0.387948 & 3.029 & 0.0900\end{array}$

FN3 $\quad-0.387948 \quad 3.029 \quad 0.0900$

FN1 $\quad-0.387948 \quad 3.029 \quad 0.0900$

$\begin{array}{llll}\text { FN2 } & -0.387948 & 3.029 & 0.0900 \\ \text { FN3 } & -0.387948 & 3.029 & 0.0900\end{array}$

$\begin{array}{rrr}1.425676 & 3.831 & 0.5850\end{array}$ $\begin{array}{lll}-0.385051 & 3.029 & 0.0900\end{array}$ $\begin{array}{lll}-0.385051 & 3.029 & 0.0900 \\ -0.385051 & 3.029 & 0.0900\end{array}$ $\begin{array}{lll}-0.385051 & 3.029 & 0.0900\end{array}$

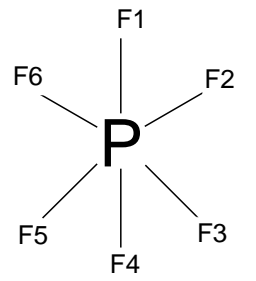

$\mathrm{CO}_{2}$

Partial Atomic Charges and Lennard-Jones Parameters

\begin{tabular}{lllll}
\hline Atom ID & Type & $\mathrm{q}_{\mathrm{i}}(\mathrm{e})$ & $\sigma(\mathrm{A})$ & $\varepsilon_{\mathrm{i}}\left(\mathrm{kcal} \mathrm{mol}^{-1}\right)$ \\
\hline $\mathrm{C}_{1}$ & $\mathrm{CCO} 2$ & 0.700000 & 2.8 & 0.0530 \\
\hline & $\mathrm{OCO} 2$ & 0.350000 & 3.05 & 0.1570
\end{tabular}

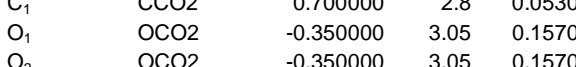
$\begin{array}{lll}-0.385051 & 3.029 & 0.0900\end{array}$ $\begin{array}{lll}-0.385051 & 3.029 & 0.0900\end{array}$ $\begin{array}{lll}-0.385051 & 3.029 & 0.0900\end{array}$ 


\section{Force Constants}

[bmim] $\left[\mathrm{PF}_{6}\right]$

Bonds

Atoms

$\mathrm{C}_{67}-\mathrm{N}_{31}$

$\mathrm{C}_{5,4}-\mathrm{N}_{1,3}$

$\mathrm{C}_{2}-\mathrm{N}_{1,3}$

$\mathrm{C}_{4}-\mathrm{C}_{5}$

$\mathrm{C}_{4,5}-\mathrm{H}_{2,3}$

$\mathrm{C}_{6,7}-\mathrm{H}_{4,5,6,7,8}$

$\mathrm{C}_{8,9}-\mathrm{H}_{9,10,11,12}$

$\mathrm{C}_{10} \mathrm{H}_{13,14,1}$

$\mathrm{C}_{8}-\mathrm{C}_{9}$

$\mathrm{C}_{9}-\mathrm{C}_{10}$

P-F

Angles

Atoms

$\frac{\text { Atoms }}{\mathrm{C}_{8}-\mathrm{C}_{7}-\mathrm{N}_{1}}$

$\mathrm{C}_{5,4}-\mathrm{N}_{1,3}-\mathrm{C}_{2}$

$\mathrm{H}_{4,5,6}-\mathrm{C}_{6}-\mathrm{N}_{3}$

$\mathrm{H}_{1}-\mathrm{C}_{2}-\mathrm{N}_{1,3}$

$\mathrm{N}_{1}-\mathrm{C}_{2}-\mathrm{N}_{3}$

$\mathrm{H}_{2,3}-\mathrm{C}_{4,5}-\mathrm{C}_{5,4}$

$\mathrm{N}_{3,1}-\mathrm{C}_{4,5}-\mathrm{H}_{2,3}$

$\mathrm{H}_{4,5,6,7,8}-\mathrm{C}_{6,7}-\mathrm{H}$

$\mathrm{H}_{7,8}-\mathrm{C}_{7}-\mathrm{C}_{8}$

$\mathrm{H}_{9,10}-\mathrm{C}_{8}-\mathrm{C}_{7}$

$\mathrm{C}^{-1} \mathrm{C}_{8} \mathrm{C}_{9}$

$\mathrm{C}_{8}-\mathrm{C}_{9}-\mathrm{C}_{10}$

$\mathrm{H}_{13,14,15}-\mathrm{C}_{10}-\mathrm{H}$

$\mathrm{C}_{8}-\mathrm{C}_{9}-\mathrm{H}$

$\mathrm{C}_{9}-\mathrm{C}_{10}-\mathrm{H}$

$\mathrm{C}_{10}-\mathrm{C}_{9}-\mathrm{H}$

$\mathrm{C}_{6,7}-\mathrm{N}_{3,1}-\mathrm{C}_{2}$
$\mathrm{C}_{6,7}-\mathrm{N}_{3,1}-\mathrm{C}_{4,5}$

F-P-F

F-P-F

$k_{\mathrm{b}}(\mathrm{kcal}$

(kcal mol ${ }^{-1}$

220.00
400.00

400.00

410.00

340.00

365.00
309.00

309.00

322.00

200.00

222.50

222.50

280.00

\begin{tabular}{l|r} 
& Dihedrals
\end{tabular}

1.4762

1.3819

1.3366
1.3610

1.3610
1.0779

1.0779
1.0775

1.0775
1.0899

1.0899

1.0954

1.0935
1.5308

1.5308
1.5373

1.5314

1.6458

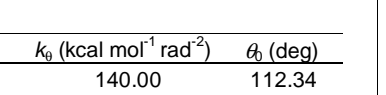

$\mathrm{C}_{2}-\mathrm{N}_{1,3} \mathrm{C}_{5,4} \mathrm{C}_{4,5}$

$\mathrm{N}_{1}-\mathrm{C}_{5}-\mathrm{C}_{4}-\mathrm{N}_{3}$

14.000

14.000
14.000

政,

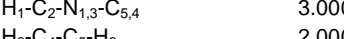

$\begin{array}{ll}\mathrm{C}_{4,5}-\mathrm{C}_{5,4}-\mathrm{N}_{1,3}-\mathrm{C}_{7,6} & 0.000\end{array}$

$\begin{array}{ll}\mathrm{C}_{4,5}-\mathrm{C}_{5,4}-\mathrm{N}_{1,3}-\mathrm{C}_{7,6} & 0.000 \\ \mathrm{H}_{2,3}-\mathrm{C}_{4,5}-\mathrm{N}_{3,1}-\mathrm{C}_{2} & 3.000\end{array}$

$\mathrm{N}_{1,3}-\mathrm{C}_{5,4}-\mathrm{C}_{4,5}-\mathrm{H}_{2,3} \quad 3.000$

$\begin{array}{ll}\mathrm{N}_{1,3}-\mathrm{C}_{2}-\mathrm{N}_{3,1}-\mathrm{C}_{6,7} & 0.000 \\ \mathrm{H}_{1}-\mathrm{C}_{2}-\mathrm{N}_{3,1}-\mathrm{C}_{6,7} & 0.000\end{array}$

$\begin{array}{ll}\mathrm{H}_{1}-\mathrm{C}_{2}-\mathrm{N}_{3,1}-\mathrm{C}_{6,7} & 0.000 \\ \mathrm{H}_{2,3}-\mathrm{C}_{4,5}-\mathrm{N}_{3,1}-\mathrm{C}_{6,7} & 0.000\end{array}$

$\mathrm{C}_{2}-\mathrm{N}_{1,3}-\mathrm{C}_{7,6}-\mathrm{H} \quad 0.195$

$\mathrm{C}_{4,5}-\mathrm{N}_{3,1}-\mathrm{C}_{6,7} \mathrm{H}-0.000$

$\mathrm{C}_{2}-\mathrm{N}_{1}-\mathrm{C}_{7}-\mathrm{C}_{8}$

$\mathrm{C}_{5}-\mathrm{N}_{1}-\mathrm{C}_{7}-\mathrm{C}_{8}$
$\mathrm{~N}_{1}-\mathrm{C}_{7}-\mathrm{C}_{8}-\mathrm{C}_{9}$

$\mathrm{H}_{11,12}-\mathrm{C}_{9}-\mathrm{C}_{10}-\mathrm{H}_{13,14,15}$

$\mathrm{C}_{8}-\mathrm{C}_{9}-\mathrm{C}_{10}-\mathrm{H}_{13,14,15}$

$\mathrm{N}_{1}-\mathrm{C}_{7}-\mathrm{C}_{8}-\mathrm{H}_{9,10}$

$\mathrm{C}_{7}-\mathrm{C}_{8}-\mathrm{C}_{9}-\mathrm{C}_{10}$

$\mathrm{H}_{7,8}-\mathrm{C}_{7}-\mathrm{C}_{8}-\mathrm{H}_{9,10}$

$\mathrm{C}_{9}-\mathrm{C}_{8}-\mathrm{C}_{7}-\mathrm{H}_{7,8}$
$\mathrm{H}_{11,1,2}-\mathrm{C}_{9}-\mathrm{C}_{8}-\mathrm{C}_{7}$

$\mathrm{H}_{9,10}-\mathrm{C}_{8}-\mathrm{C}_{9}-\mathrm{H}_{11,12}$

$\mathrm{H}_{9,10}-\mathrm{C}_{8}-\mathrm{C}_{9}-\mathrm{C}_{10}$

Impropers

\begin{tabular}{lcc}
\multicolumn{1}{c}{ Impropers } & & \\
\hline Atoms & $k_{W}\left(\mathrm{kcal} \mathrm{mol}^{-1} \mathrm{rad}^{-2}\right)$ & $\psi_{0}(\mathrm{deg})$ \\
\hline $\mathrm{C}_{2}-\mathrm{N}_{1}-\mathrm{N}_{2}-\mathrm{H}_{1}$ & 0.500 & 0 \\
$\mathrm{~N}_{1,3}-\mathrm{C}_{5,4}-\mathrm{C}_{2}-\mathrm{C}_{7,6}$ & 0.600 & 0 \\
$\mathrm{C}_{4,5}-\mathrm{C}_{5,4}-\mathrm{N}_{3,1}-\mathrm{H}_{2,3}$ & 0.500 & 0
\end{tabular}

108.43

111.62

109.47

125.67

90.00

180.00
Force Constants

[bmmim] $\left[\mathrm{PF}_{6}\right]$

\begin{tabular}{|c|c|c|c|c|c|c|}
\hline \multicolumn{3}{|l|}{ Bonds } & \multicolumn{2}{|l|}{ Dihedrals } & \multirow[b]{2}{*}{$n$} & \multirow[b]{2}{*}{$\delta(\mathrm{deg})$} \\
\hline Atoms & $k_{\mathrm{b}}\left(\mathrm{kcal} \mathrm{mol}{ }^{-1} \AA^{-2}\right)$ & $r_{0}(\AA)$ & Atoms & $k \chi\left(\mathrm{kcal} \mathrm{mol}^{-1}\right)$ & & \\
\hline$\overline{\mathrm{C}_{2}-\mathrm{C}_{11}}$ & 229.63 & 1.4873 & $\mathrm{C}_{11}-\mathrm{C}_{2}-\mathrm{N}_{1,3}-\mathrm{C}_{5,4}$ & 3.000 & 2 & 180 \\
\hline \multirow{2}{*}{$\mathrm{C}_{11}-\mathrm{H}_{1,16,17}$} & 322.00 & 1.0915 & $\mathrm{C}_{11}-\mathrm{C}_{2}-\mathrm{N}_{1,3}-\mathrm{C}_{7,6}$ & 0.000 & 2 & 180 \\
\hline & & & $\mathrm{N}_{1,3}-\mathrm{C}_{2}-\mathrm{C}_{11}-\mathrm{H}_{1,16,17}$ & 0.195 & 2 & 180 \\
\hline \multicolumn{3}{|l|}{ Angles } & & & & \\
\hline Atoms & $k_{\theta}\left(\mathrm{kcal} \mathrm{mol}^{-1} \mathrm{rad}^{-2}\right)$ & $\theta_{0}(\mathrm{deg})$ & Improper & & & \\
\hline $\mathrm{C}_{11}-\mathrm{C}_{2}-\mathrm{N}_{1,3}$ & 45.80 & 126.23 & Atoms & $k_{\psi}\left(\mathrm{kcal} \mathrm{mol}^{-1} \mathrm{rad}^{-2}\right)$ & $\psi_{0}(\mathrm{deg})$ & \\
\hline $\mathrm{C}_{2}-\mathrm{C}_{11}-\mathrm{H}_{1,16,17}$ & 33.43 & 111.08 & $\mathrm{C}_{2}-\mathrm{N}_{1}-\mathrm{N}_{3}-\mathrm{C}_{11}$ & 0.500 & 0 & \\
\hline $\mathrm{H}-\mathrm{C}_{11}-\mathrm{H}$ & 35.50 & 107.81 & & & & \\
\hline
\end{tabular}

\section{Force Constants}

$\mathrm{CO}_{2}$

\begin{tabular}{|c|c|c|}
\hline & & \\
\hline Atoms & $k_{\mathrm{b}}\left(\mathrm{kcal} \mathrm{mol} \mathrm{l}^{-1} \AA^{-2}\right)$ & $r_{0}(\AA ̊)$ \\
\hline
\end{tabular}

\begin{tabular}{lcc} 
Angle & & \\
\hline Atoms & $k_{\theta}\left(\mathrm{kcal} \mathrm{mol}^{-1} \mathrm{rad}^{-2}\right)$ & $\theta_{0}(\mathrm{deg})$ \\
\hline $\mathrm{O}_{1}-\mathrm{C}_{1}-\mathrm{O}_{2}$ & 56.00 & 180.00
\end{tabular}

Notes on assignment of CHARMM atom types:

itrogens are in imidazolium, therefore NR2 is used as NR1

FN1, FN2, FN3 are defined as orthogonal atoms such that two fluorines of the same type form a $180^{\circ}$ angle.

New carbon $\mathrm{C} 11$ in $[\mathrm{bmmim}]\left[\mathrm{PF}_{6}\right]$ was defined as $\mathrm{C} 5 \mathrm{M}$ (CN9), which represents a methyl group next to an aromatic ring.

C8 \& C9 were used as CN8 and its hydrogens as HN8 when having

intramolecular parameters that had imidazole atoms within

Dihedral parameter for the butyl chain is taken as CT2-CT2-CT2-CT3 